\section{Validez de los estudios de asociación entre geohelmintos e incidencia de malaria: ¿Debería impactar las políticas de salud?}

\section{Validity of studies on the association between soil-transmitted helminths and the incidence of malaria: Should it impact health policies?}

\section{Julián Alfredo Fernández ${ }^{1}$}

Álvaro Javier Idrovo²

\section{Zulma Milena Cucunubá1,3}

\section{Patricia Reyes $^{1}$}

1 Departamento de Salud Pública. Facultad de Medicina. Universidad Nacional de Colombia. Bogotá, Colombia.

${ }^{2}$ Centro de Investigación de Sistemas de Salud. Instituto Nacional de Salud Pública de México. Cuernavaca, México.

${ }^{3}$ Grupo de Parasitología. Instituto Nacional de Salud. Bogotá, Colombia.

\section{Resumen}

Introducción: La distribución mundial de las geohelmintiasis y la malaria se encuentra ampliamente sobrepuesta. Algunos estudios sugieren una asociación entre las infecciones por geohelmintos y la incidencia de malaria. Objetivos: Identificar la evidencia epidemiológica disponible y evaluar la validez de estos estudios. Metodología: Una revisión sistemática fue realizada en bases de datos especializadas. Los estudios identificados fueron analizados críticamente y ordenados según clasificación de la U.S. Preventive Services Task Force. Se identificaron las principales limitaciones metodológicas de cada estudio. Resultados: Se encontraron seis estudios publicados sobre el tema. Solo dos estudios tienen un alto nivel de evidencia (nivel I), tres de nivel II-2, y uno de nivel III-3. Existen importantes limitaciones metodológicas para aclarar la asociación entre geohelmintos e incidencia de malaria. Conclusiones: Es apresurado discutir las potenciales implicaciones en salud pública de estos hallazgos dada la escasez de estudios y la validez limitada de la evidencia existente. Futuros estudios con nuevas consideraciones metodológicas podrían mejorar el conocimiento acerca de esta asociación. Sin embargo, es más importante realizar acciones sobre los determinantes estructurales para controlar y prevenir la ocurrencia de ambas enfermedades.

Palabras clave: Helmintos. Malaria. Comorbilidad. Sesgo (epidemiología). Control de enfermedades transmisibles. 


\section{Abstract}

Introduction: The global distribution of malaria and soil-transmitted helminths is widely overlapped. Some studies suggest a possible association between helminth infection and incidence of malaria. Objectives: To identify the available epidemiologic evidence and to assess the validity of these studies. Methods: A systematic review was carried out in specialized databases. The studies identified were critically analyzed and ranked according to the U.S. Preventive Services Task Force's classification. The major methodological limitations of each study were identified. Results: Six studies on the topic were found. Only two studies had a high evidence level (level I), three had level II-2, and one had level III-3. There are important methodological limitations for clarifying the association between soiltransmitted helminths and the incidence of malaria. Conclusion: It is too early to discuss the potential public health implications of these findings, given the lack of studies and limited validity of the evidence available. Further studies with new methodological considerations could improve the knowledge on the association. However, it is more important to carry out actions on structural determinants to control and prevent the occurrence of both diseases.

Keywords: Helminths. Malaria. Comorbidity. Bias (epidemiology). Communicable disease control.

\section{Introducción}

Se estima que en el mundo más de 2000 millones de personas están infectadas por geohelmintos $^{1,2}$, la mayoría de las cuales vive en países en vía de desarrollo donde la malaria tiene altas incidencias. Esta enfermedad presenta aproximadamente entre 300 a 500 millones de casos cada año y es responsable de más de dos millones de muertes en el mismo periodo ${ }^{3}$. Debido a las características propias de la transmisión de estas infecciones, su distribución se encuentra sobrepuesta en amplias regiones y una gran proporción de la población mundial sufre coinfección por estos parásitos, ocasionando importantes efectos sobre la comorbilidad, especialmente la anemia multifactorial ${ }^{4}$.

En 1978 se sugirió por primera vez que, además de la comorbilidad, podría existir una asociación entre la infección por geohelmintos y la incidencia de malaria clínica ${ }^{5}$. Sin embargo, la mayoría de los estudios sobre estas asociaciones han sido adelantados solamente en los últimos 10 años. Estos trabajos también han explorado un potencial efecto protector de los geohelmintos para el desarrollo de malaria complicada ${ }^{6}$. A pesar del renovado interés en este tema, las investigaciones siguen siendo muy escasas y sus resultados divergentes. Aunque se han sugerido varias hipótesis evolutivas e inmunológicas, los mecanismos que sustentarían estas potenciales asociaciones permanecen todavía desconocidos?

Algunos autores han llamado la atención sobre las limitaciones metodológicas y la confusión presente en estas investigaciones ${ }^{7,8}$. Aunque los geohelmintos y la malaria tienen diferentes mecanismos de transmisión, comparten determinantes ambientales, culturales, socioeconómicos, conductuales y biológicos propios del hospedero, que podrían actuar como potenciales variables confusoras. También se ha señalado la probable influencia del sesgo de selección y las limitaciones de validez intrínseca de estas investigaciones, lo que se explica en parte porque muchos datos son tomados de estudios realizados con otros objetivos ${ }^{9}$. La 
mayoría de las revisiones se centran en describir los potenciales mecanismos biológicos que sustentan la asociación y en enumerar las evidencias epidemiológicas que soportarían la relación. Debido a las importantes repercusiones que estos resultados podrían tener sobre las políticas de Salud Pública, especialmente sobre los programas de control de malaria y la determinación de acciones prioritarias en salud pública ${ }^{10}$, el análisis epidemiológico crítico y sistemático de los estudios se hace indispensable.

Dentro de la epidemiología, el estudio de la comorbilidad ha tenido un importante auge en los últimos años, asociado especialmente con el envejecimiento de la población, y sus importantes repercusiones en los gastos en salud ${ }^{11}$. Con este término se hace referencia a la presencia de una o más enfermedades en un individuo que tiene una "enfermedad índice"12. En nuestro caso la geohelmintiasis podría considerarse como enfermedad índice, ya que según la hipótesis sugerida se presenta primero que la malaria. La relación entre la infección con geohelmintos y la incidencia de malaria resulta ser un tipo de comorbilidad no frecuentemente estudiada por varias razones: a) se presenta entre dos enfermedades infecciosas, b) ocurre predominantemente entre menores de edad ${ }^{11}, \mathrm{yc}$ ) no hay claridad sobre la relación entre malnutrición y malaria, pues a pesar de que estudios contradictorios han postulado que la malnutrición protege de la malaria ${ }^{13}$; el estado nutricional se convierte en una variable intermedia en la relación que debe ser tratada como tal durante el análisis estadístico. Debido a la complejidad de este tema, este trabajo explora la validez de las investigaciones hechas sobre la asociación de las geohelmintiasis con la incidencia de malaria, y propone discutir si es necesario investigar más sobre el tema $\mathrm{o}$, si por el contrario, se deben tomar medidas directamente para prevenir su ocurrencia.

\section{Metodología}

Utilizando la base de datos Pubmed, se buscaron artículos originales que exploraran la asociación entre geohelmintiasis y la incidencia de malaria. Se utilizaron como palabras de búsqueda las siguientes combinaciones "helmintos" y "malaria", "Ascaris" y "malaria", "Trichuris" y "malaria", "hookworm" y "malaria", "helmintos" y "severe malaria", "Ascaris" y "severe malaria", "Trichuris" y "severe malaria", y "hookworm" y "cerebral malaria". Adicionalmente se hizo búsqueda dentro de las referencias de los artículos de revisión publicados en los últimos 3 años $(n=15)$ sobre el tema.

Dos observadores, de manera independiente, revisaron el título y en caso necesario el resumen de todas las publicaciones obtenidas en la búsqueda. Se incluyeron en este análisis aquellos estudios originales relacionados directamente con la pregunta de interés. Se excluyeron los trabajos sobre otras asociaciones de geohelmintos con anemia severa, infecciones múltiples o infecciones mixtas. Tampoco se tuvieron en cuenta las investigaciones realizadas en grupos de embarazadas, ni aquellas con otros helmintos como Schistosoma sp.

Los estudios fueron ordenados según nivel de evidencia, usando la clasificación de la U.S. Preventive Services Task Force. Esta tiene las siguientes categorías: I. Evidencia obtenida de ensayo clínico aleatorizado; II-1. Evidencia obtenida de estudio bien diseñado sin aleatorización; II-2. Evidencia obtenida de estudio de cohorte o casos y controles bien diseñados, preferiblemente de más de un centro o grupo de investigación; II-3. Evidencia obtenida de múltiples series de tiempo con o sin intervención, y III. Opiniones de autoridades respetadas, basadas en la experiencia, estudios descriptivos y reportes de caso, o reportes de comités de expertos ${ }^{14}$.

\section{Resultados}

Solamente se han publicado seis estudios que evalúan la asociación entre la infección por geohelmintos y la incidencia de malaria. Un resumen de los diseños se presenta en la Tabla 1. 


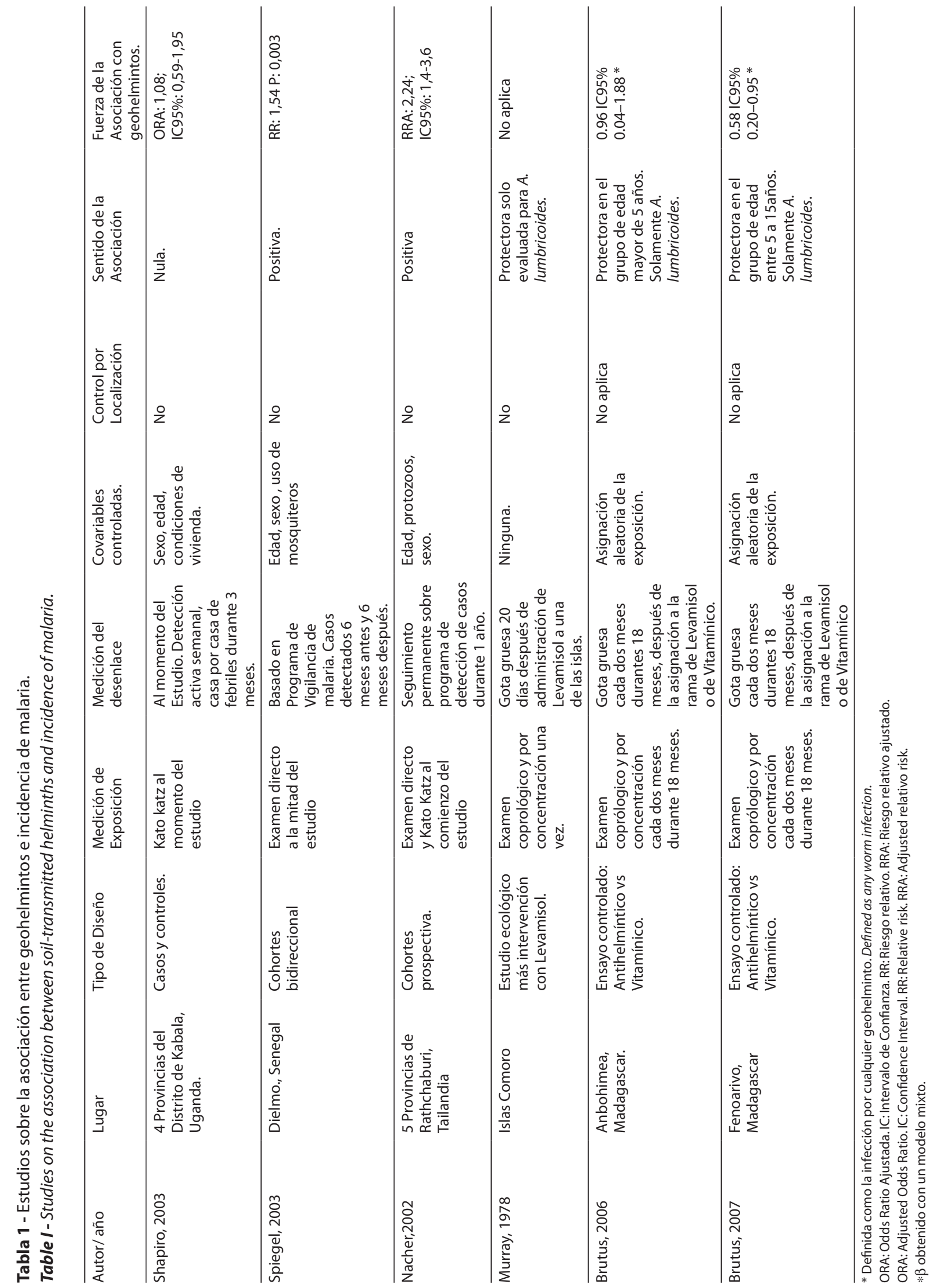




\section{Nivel de evidencia I}

El estudio de Brutus y colaboradore ${ }^{15} \mathrm{de}$ 2003, fue el primero en usar un diseño aleatorizado. Este trabajo exploró la interacción entre la disminución de la prevalencia de Ascaris lumbricoides y su carga parasitaria, con la densidad de infección por Plasmodium falciparum en una región endémica de Madagascar. Para tal fin usaron datos de un ensayo clínico en el que se comparó el tratamiento con levamisol con un multivitamínico. Mediante un seguimiento de 16 meses se pudieron evidenciar simultáneamente los ciclos de infección-reinfección con geohelmintos y los casos incidentes de malaria. Los resultados, además de mostrar que el levamisol es muy efectivo contra $A$. lumbricoides, evidenciaron - aunque solamente entre los mayores de cinco años de edad - que el grupo asignado al tratamiento con levamisol incrementó progresivamente las densidades de infección por P. falciparum comparado con el grupo control ${ }^{15}$. De esta manera, los resultados de este estudio sugieren indirectamente un potencial efecto protector de A. lumbricoides contra el desarrollo de malaria. El hecho de que la asociación no haya sido encontrada en el grupo de edad de menores de cinco años de edad, puede ser explicado en razón a que en este país la malaria es mesoéndemica, y se necesitan varios años para adquirir la inmunidad para la malaria como también un potencial equilibrio entre los parásitos ${ }^{15}$. Por tratarse de un ensayo clínico aleatorizado, se espera que los resultados no estén afectados por confusión. Los hallazgos de este estudio y otro ensayo clínico realizado por el mismo grupo, reproducen los encontrados por Murray, en 1978, y difieren de los resultados obtenido por los estudios observacionales analíticos realizados sobre el tema. Sin embargo, no se puede descartar que los resultados puedan estar afectados por el sesgo de localización (que se discutirá más adelante), a pesar de que el estudio fue realizado en una misma localidad.

Adicionalmente, si se tiene en cuenta que el levamisol es un antihelmíntico con un importante efecto inmunomudulador, usado en terapia de cáncer y enfermedades autoinmunes ${ }^{16}$, se pondría en duda si los hallazgos obtenidos en este estudio se relacionan directamente con la infección por A. lumbricoides ó son un efecto secundario del levamisol, así que podrían eventualmente obtenerse diferentes resultados si el medicamento antihelmíntico utilizado hubiera sido otro. Incluso algunos autores han sugerido que el levamisol tiene un efecto sobre el secuestro de Plasmodium spp y por lo tanto, un potencial uso para malaria complicada $^{17}$, por lo que también por esta otra vía este medicamento podría afectar la incidencia de malaria.

En otro estudio más reciente, publicado también por Brutus y colaboradores ${ }^{18}$ realizado en una región con transmisión de Schistosoma mansoni a 1250 metros sobre el nivel del mar (msnm), donde fueron frecuentes las fumigaciones de los hogares con DDT entre 1993 y 1998, y muy aledaña a la estudiada en el estudio aleatorizado descrito antes ${ }^{15}$, se comparó también el efecto de dar tratamiento con levamisol o con multivitamínicos sobre la incidencia de malaria no complicada mediante un ensayo clínico aleatorizado con un diseño muy similar al adelantado por este mismo grupo de trabajo. Los resultados después de 18 meses, obtenidos mediante modelos de efectos fijos y aleatorios, mostraron que quienes fueron tratados con levamisol y conseguían disminución de la carga de huevos de $A$. lumbricoides presentaron aumentos de la densidade de infección por P. falciparum, pero este efecto se observó solamente entre los sujetos con edades entre 5 y 14 años. Como se puede apreciar, los resultados son muy similares a los descritos previamente y las limitaciones de la evidencia aportada por este trabajo son similares al anterior.

\section{Nivel de evidencia II-2}

En 1998, Nacher y colaboradores ${ }^{19}$ llevaron a cabo un estudio de cohortes prospectivo en cinco veredas tailandesas. Este estudio consistió en una encuesta basal 
de parasitismo intestinal, y un seguimiento de un año para evaluar la incidencia de malaria. Basándose en el antecedente de un programa educativo sobre malaria ya implementado, los autores buscaron garantizar que todos los casos sintomáticos consultaran al único centro de diagnóstico y distribución del tratamiento de la región. Este trabajo encontró una asociación positiva entre las infecciones por geohelmintos y la incidencia de malaria por Pfalciparum. Los autores presentaron los resultados ajustados por sexo y edad. Sin embargo, el estrato socioeconómico, el nivel educativo, las condiciones de las viviendas no fueron tenidas en cuenta, por lo que pudieron actuar como potenciales variables confusoras. Tampoco se tiene en cuenta la localización o la procedencia de los sujetos de estudio; dado que procedían de cinco localidades diferentes, el estudio pudo incurrir en un sesgo de localización.

Shapiro y colaboradores en 2003 condujeron un estudio de casos y controles en cuatro provincias rurales del distrito de Kabala, Uganda ${ }^{20}$. La selección de los casos, definida como individuo con resultado de gota gruesa confirmada para Plasmodium $s p$, se realizó mediante un programa de vigilancia semanal de malaria, en el cual los promotores de salud visitaron cada vivienda en búsqueda de sintomáticos. Los controles fueron habitantes no sintomáticos de los mismos distritos. Un detalle que puede pasar desapercibido en este trabajo es que para el análisis adelantado solo fueron incluidos los casos provenientes de dos de las provincias, Kikuto y Rwandamira, mientras que los controles provenían de estas provincias y adicionalmente de Kabirizi y Kigara. Los autores justifican este diseño en la dificultad de reclutar casos por la migración en las provincias de Kabirizi y Kigara. Según resultados presentados en el mismo artículo, las dos poblaciones de las cuales provenían los casos tenían significativamente mayores prevalencias de geohelmintiasis que las otras dos poblaciones. Por esta razón los casos pudieron tener una mayor probabilidad de estar expuestos a los geohelmintos que los controles (sesgo de selección). De esta manera, los resultados tenderían a ser una sobreestimación de la asociación y podrían explicar que la asociación no haya sido detectada. Infortunadamente, en el artículo no se presentan los análisis exclusivamente con controles provenientes de las dos provincias donde fueron reclutados los casos. Una de las ventajas de este estudio es que se realizó control de covariables como estrato socioeconómico y condiciones de las viviendas (piso, techo, saneamiento) utilizando el índice compuesto desarrollado por Filmer ${ }^{21}$. La Odds Ratio (OR) cruda no es presentada por los autores es su manuscrito. Es llamativo, sin embargo, que al calcularla (OR: 0,87; Intervalo de Confianza 95\%: 0,58$1,29)$, ésta no difiere significativamente de la Odds Ratio ajustada presentada por los investigadores en su trabajo (OR: 1,08: IC94\%: 0,59-1,95).

El hallazgo presentado anteriormente puede tener tres explicaciones. Primero, que las condiciones de vivienda y el estrato socioeconómico no son variables confusoras de la asociación entre geohelmintos y malaria. Segundo, que se trate de un problema de confusión residual, y no se haya detectado el papel de otras covariables extrañas no medidas como la baja escolaridad, la desnutrición o la localización. Tercero, que la influencia de las variables confusoras medidas en el estudio no haya sido adecuadamente detectada. Es probable, por ejemplo, que el Índice de Filmer, por tratarse de un indicador compuesto y complejo, que consolida numéricamente a un conglomerado de malas condiciones de vivienda, no tenga la especificidad necesaria para detectar determinados patrones específicos de infraestructura de la casa. Por ejemplo, el piso de tierra sin ventanas que es un factor de riesgo común para geohelmintos y malaria. Otra limitación de los resultados de este trabajo es que no fue posible diferenciar la especie de Plasmodium spp. Los autores sustentan que de acuerdo a datos previos, la especie más predominante en el distrito es $P$. falciparum.

Otra cohorte, esta vez bidireccional, fue 
estudiada en Dielmo, Senegal, en 1998 por Spiegel y colaboradores ${ }^{22}$. En este trabajo los investigadores realizaron examen coprológico a 80 niños de la población. Las covariables de la población de estudio y su comparación con las de la población marco no son presentadas tampoco en el manuscrito. Al igual que estudios previos, Spiegel condujo su estudio sobre la base de un programa de vigilancia de malaria, determinando la ocurrencia de casos de malaria en los seis meses previos y posteriores a la medición de la exposición. Se encontró una mayor incidencia de malaria en los infectados con geohelmintos. En este trabajo fueron tenidas en cuenta como covariables de ajuste la edad, el sexo y el uso de toldillos, pero no se consideraron variables socioeconómicas.

\section{Nivel de evidencia III-3}

Murray $^{5}$, en 1978, mientras adelantaba estudios de malaria en dos islas del complejo Comoro, observó que en la isla con mayor prevalencia de A. lumbricoides $(93 \%)$ había menor prevalencia de malaria $(1,7 \%)$ mientras que en la otra isla que tenía la más baja prevalencia de este geohelminto (24\%) la prevalencia de malaria era mayor $(23 \%)$. Este autor sugirió, a partir de estas observaciones, que existía una relación negativa entre la prevalencia de $A$. lumbricoides y $P$. falciparum, postulando por primera vez una asociación protectora entre la infección por geohelmintos y malaria.

Murray completó sus observaciones al año siguiente, llevando a cabo un ensayo placebo controlado en las mismas poblaciones, encontrando que la población asignada a tratamiento con antihelmíntico presentó un incremento significativo de la incidencia de malaria, 20 días después de la intervención, comparada con el grupo placebo controlado. Las principales limitaciones de estas observaciones comprenden el limitado número de observaciones y el corto seguimiento del desenlace, ya que cambios en la incidencia de malaria en menos de un mes pueden ser explicados por pequeños brotes introducidos o por la estacionalidad. Adicionalmente, a excepción de la edad, ninguna covariable fue tenida en cuenta; la desnutrición que, como se sabe, es más prevalente en los infectados con geohelmintos, pudo actuar como una importante variable confusora, siendo bien conocidas, aunque controvertidas, las relaciones entre malaria y desnutrición. En el trabajo adelantado por este autor, no se tuvo en cuenta tampoco que el patrón de distribución de ambos parásitos podría obedecer a condiciones ecológicas y no hay duda que exista una relación biológica directa entre ellos. Sin embargo, aún con todas las limitaciones mencionadas es inte $\neg$ resante que los resultados de Murray hayan sido consistentes con lo encontrado por los únicos dos ensayos clínicos controlados publicados. Además, algunos autores parecen sugerir que los resultados de Murray son consistentes con aquellos que sugieren un efecto protector de los geohelmintos para el desarrollo de malaria complicada ${ }^{6}$.

\section{Discusión}

Los estudios existentes sobre la asociación entre infección por geohelmintos y la incidencia de malaria, analizados en esta revisión, son todavía escasos y sus resultados altamente divergentes. En resumen, dos estudios de cohortes cerradas, encontraron una asociación positiva entre la infección con geohelmintos y malaria ${ }^{19,22}$, uno de casos y controles, encontró una asociación nula ${ }^{20} \mathrm{y}$ otros tres: uno ecológi$\operatorname{co}^{5}$ y dos ensayos clínicos aleatorizados ${ }^{15,18}$ sobre el tema encontraron una asociación protectora. El hecho de que los resultados encontrados en los ensayos controlados de Brutus y colaboradores no sean consistentes con ninguno de los estudios analíticos realizados podría ser explicado por las limitaciones metodológicas de estos estudios, especialmente por la potencial influencia de la confusión.

Si bien los geohelmintos y la malaria tienen mecanismos de transmisión diferentes, comparten determinantes sociales y ambientales de su ocurrencia, de manera 
que antes de una asociación biológica real entre ambos parásitos, la influencia de los codeterminantes de ambas enfermedades debería ser analizada (Gráfica 2). Así por ejemplo, la asociación encontrada por Nacher y Spiegel ${ }^{19,22}$, podría explicarse simplemente porque las poblaciones infectadas por geohelmintos tienen mayor riesgo de enfermar debido a determinantes estructurales (pobreza, desnutrición, baja escolaridad) que la población no infectada con geohelmintos. Dicho de otra manera, que las poblaciones en mayor riesgo de infección por geohelmintos tienden a ser las mismas en mayor riesgo de malaria en las regiones donde ambas enfermedades son endémicas. Las evidencias sobre esta superposición de enfermedades, si bien son conocidas desde hace varias décadas, sólo recientemente han empezado a ser exploradas con técnicas específicas de la epidemiología espacial, que han puesto de manifiesto la importancia de conocer los contextos en los cuales se encuentran los individuos en riesgo ${ }^{23}$. Algunas de estas potenciales variables son presentadas y discutidas a continuación.

Condición de la vivienda: Las malas características de la vivienda tales como la ausencia de herramientas de contención para la entrada de insectos (puertas, angeos o ventanas), el tipo de techo o el material de paredes son determinantes de riesgo bien conocidos para la infección por malaria ${ }^{24}$. Se ha comprobado también que habitar casas con pobre infraestructura física (de madera o palma) tiene un riesgo mayor para desarrollo de malaria que vivir en casas con buenos materiales de construcción ${ }^{25}$. Este riesgo aparentemente se incrementa si la vivienda está cerca a una fuente de agua ${ }^{26}$. Otras condiciones de la casa, tales como el piso de tierra, han sido asociadas con presencia de geohelmintiasis ${ }^{27}$. Así, en zonas de malas condiciones socioeconómicas se comparten condiciones de vivienda que

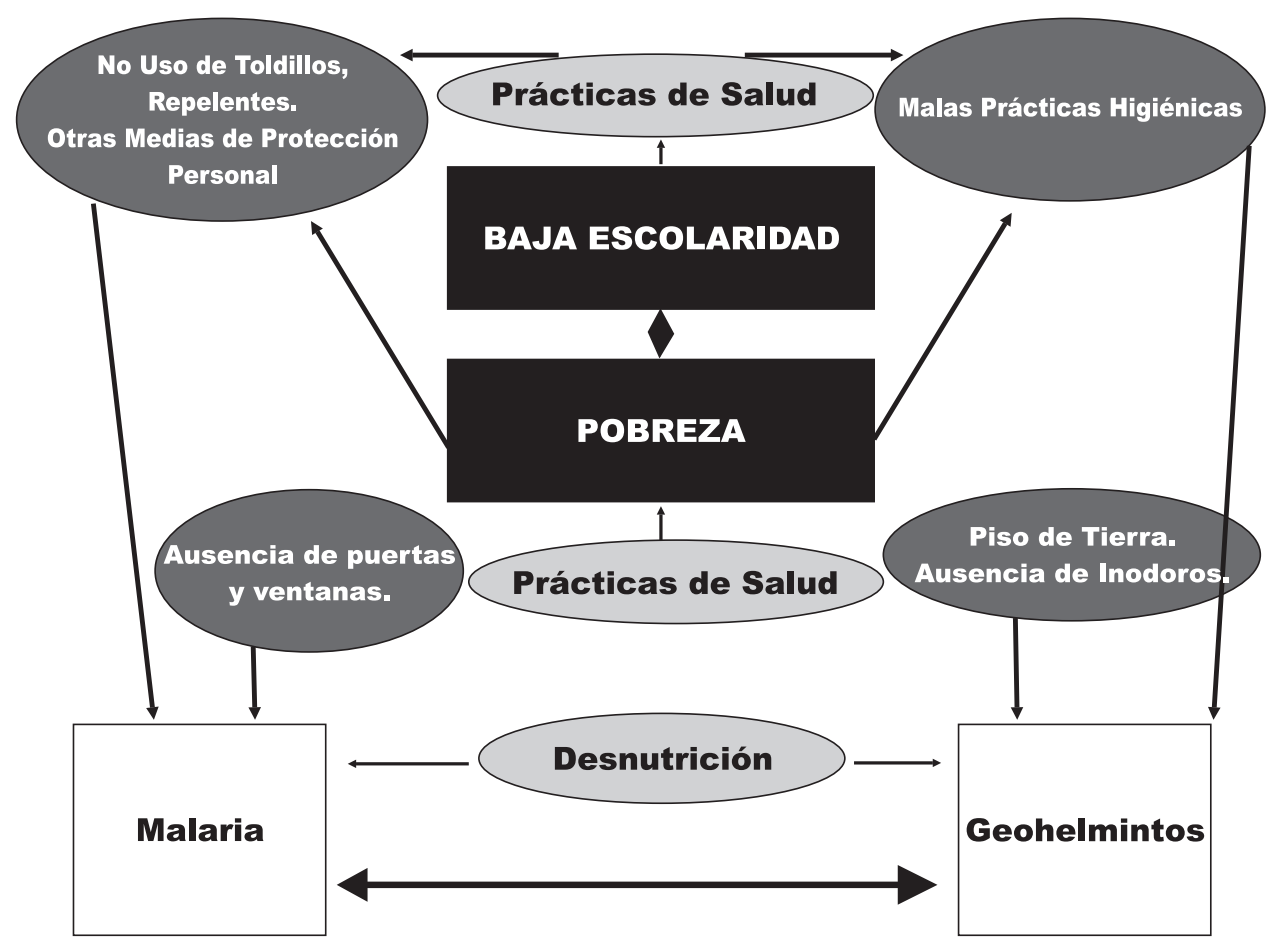

Gráfica 1 - Redes causales entre la ocurrencia de geohelmintos y malaria. Determinantes estructurales. Determinantes de anclaje. $\square$ Factores de Riesgo.

Figure 1 - Causal networks between soil-transmitted helminths and malaria occurrence.

Structural determinants. Anchorage determinants. $\square$ Risk Factors. 
son riesgosas para ambas patologías, lo cual por sí mismo genera mayor incidencia tanto de malaria como de geohelmintiasis, independientemente de una relación biológica entre ambos parásitos. Esta covariable debe ser considerada en los estudios sobre el tema, pero deconstruida, de manera que se mida en cada sujeto de estudio las características del techo, el piso y las paredes de la vivienda en que habita. De este modo se identificarían patrones de vivienda que podrían actuar como potenciales variables confusoras, por ser factores de riesgo común para geohelmintos y malaria.

Desnutrición: En los niños, la desnutrición crónica lleva a la alteración del desarrollo del timo y por lo tanto a un déficit de la producción y la maduración de los linfocitos $\mathrm{T}$, ocasionando defectos inmunes graves como la leucopenia, que genera por sí sola una mayor susceptibilidad a padecer cualquier infección ${ }^{28,29}$. Los niños malnutridos son deficientes en IgE protectora contra $A$. lumbricodes ${ }^{30} \mathrm{y}$ se ha descrito que padecen una alteración de la regulación en la inmunidad a helmintos ${ }^{31}$. Por su parte, algunos estudios han encontrado una asociación entre deficiencias nutricionales y bajo peso con incidencia de malaria ${ }^{32,33 .}$ Es decir, la desnutrición crónica también actuaría como un factor de riesgo común para ambas enfermedades.

Sin embargo, desde otro punto de vista es probable que el defecto nutricional producido por las geohelmintiasis pudiera generar mayor riesgo de episodios maláricos, configurando así un mecanismo no inmunológico de la asociación entre ambos parásitos que hasta ahora no ha sido discutido. Es decir que la desnutrición asociada con las geohelmintiasis genera menor competencia inmune y por lo tanto mayor riesgo de infecciones. Así, el estado nutricional del hospedero determina la respuesta a la infección, pero igualmente la infección puede ser causa de la desnutrición.

Sin embargo, la asociación entre geohelmintiasis y malaria podría también estar confundida en sentido contrario, dado que estos primeros parásitos se asocian a la desnutrición crónica y que existe cierta evidencia controvertida de que la desnutrición podría proteger para malaria ${ }^{5,13}$.

Actividad económica: La actividad laboral en zonas endémicas de malaria, tal como la agricultura, ha sido asociada con mayor riesgo de malaria en especial en zonas rurales ${ }^{34}$. Igualmente, estudios han demostrado mayor riesgo de geohelmintiasis en personas que desarrollan actividades de agricultura, por el contacto permanente con la tierra ${ }^{35}$.

Uso de medidas de protección para malaria: Prácticas tales como el uso de toldillos, e incluso el uso de hamacas impregnadas ${ }^{33}$ y otras como el tipo de techo de las casas, son unas de las medidas de protección más importantes para prevención de malaria ${ }^{36}$. Estas prácticas están directamente relacionadas con la educación, la cultura y la disponibilidad. Es probable que las personas que hacen poco uso de estas medidas de protección sean las mismas con deficientes prácticas sanitarias y poca disponibilidad de servicios básicos, lo cual genera riesgo de geohelmintiasis.

\section{Sesgo de localización}

Ignorar la procedencia de los sujetos de estudio, como se ha realizado hasta ahora, podría ocasionar falsas inferencias ${ }^{37}$. Los participantes podrían provenir de diferentes microecosistemas ambientales y sociales, con diferente riesgo de malaria y geohelmintiasis, determinados por la localización frente a fuentes de riesgo ecológico para ambas enfermedades más que por una verdadera interacción inmune entre los parásitos. Se sabe, por ejemplo, que variaciones de menos de 200 msnm entre diferentes casas de una misma vereda se han relacionado con diferencias significativas en el riesgo de malaria ${ }^{33}$ y de la misma manera, de acuerdo a cambios en la disponibilidad de servicios públicos domiciliarios o aguas residuales en una misma localidad, la prevalencia de geohelmintiasis puede tener patrones de distribución con áreas de mayor concentración. El sesgo de localización aparece 
cuando no se tiene en cuenta la procedencia de los sujetos de estudio, y la probabilidad de estar coinfectado con geohelmintos y malaria sea potencialmente influida por esta localización, en el marco de una localidad con distribución heterogénea de fuentes de riesgo para ambas enfermedades. El problema se maximizaría en los análisis con datos procedentes de varias localidades; futuros estudios podrían indagar el papel de esta covariable.

\section{Plausibilidad biológica de la interacción helmintos-malaria y su maleabilidad}

Existen algunas teorías inmunológicas sustentadas en evidencia experimental, especialmente en modelos murinos, que podrían respaldar la asociación de las helmintiasis con episodios de malaria. Se sabe que la infección por $P$. falciparum induce una respuesta Thl proinflamatoria caracterizada por aumento de TNF $\alpha$ e INF $\lambda$, la cual es responsable de la eliminación de la carga parasitaria en hepatocitos y en eritrocitos y además limita la replicación del parásito ${ }^{38}$. Por su parte, los helmintos generan una respuesta inmune polarizada hacia Th2, con inducción de citoquinas IL4, IL-5 e IL$7^{39}$. La respuesta Th1 parece regularse por la Th2 con IgE y TGF . Así, se ha sugerido que en presencia de helmintos la respuesta Th2 inhibe el papel antiparasitario de la respuesta proinflamatoria Thl y puede generar en el hospedero mayor susceptibilidad a infección malárica. Esta inmunomodulación de la respuesta inflamatoria por helmintos podría corresponder a una forma de defensa del hospedero como adaptación evolutiva entre los parásitos y la malaria?

En malaria, la inmunidad protectora es dependiente de anticuerpos, pero la adquisición de este tipo de inmunidad es lábil y dependiente de la intensidad y duración de la exposición parasitaria. Así, en presencia de una respuesta polarizada $\mathrm{Th} 2$, el medio ambiente de citoquinas en IL-4 e IL-3 estimula la producción de IgG4 e IgE ${ }^{40}$, los cuales son anticuerpos no citofílicos, con disminución de anticuerpos citofílicos
(IgG1 e IgG3), los cuales se han encontrado que son los principales efectores de la inmunidad adquirida en malaria ${ }^{37}$.

Es interesante analizar que existen también mecanismos biológicos que podrían sustentar una asociación opuesta, es decir, protectora entre la infección con geohelmintos y la incidencia de malaria. Así por ejemplo, los hallazgos de Brutus y colaboradores pueden sustentarse en base a algunos estudios donde se analiza un posible efecto inmunomodulador de la infección por Plasmodium y Schistosoma como consecuencia de la reactividad cruzada de antígenos compartidos entre los dos parásitos, la cual podría aparentemente generar una mayor capacidad para la respuesta IgG3 favoreciendo la eliminación de Plasmodium spp en los infectados con Schistosoma ${ }^{41,42}$. Estos hallazgos son principalmente con Schistosoma, y aún preliminares, pero evidencian cómo es posible encontrar mecanismos biológicos que sustenten una asociación y aún así la plausibilidad no es criterio suficiente.

Por lo anterior, pese a estas posibilidades que permite la biología para entender la interacción geohelmintos-malaria, el estudio epidemiológico de estos mecanismos biológicos resulta todavía ser un gran desafío ${ }^{43}$. En general, se pueden observar dos aproximaciones; una cuando la interacción ocurre entre dos o más factores de riesgo, y otra cuando la interacción es entre dos eventos en salud. El primer caso hace referencia explícita a los conceptos de modificación de efecto e interacción en epidemiología ${ }^{44}$, mientras que el segundo hace referencia a comorbilidad ${ }^{12}$. Dado que la interacción geohelmintos-malaria ocurre entre eventos, una consecuencia directa es que el abordaje epidemiológico debería usar las técnicas explícitas para tal fin. Es recomendable usar medidas de comorbilidad como los rho obtenidos con modelos de regresión probit bivariados ${ }^{10}, \mathrm{o}$ adaptar índices ya conocidos como el Cumulative Illness Rating Scale, la Clasificación Kaplan-Feinstein, el Índice Charlson de Comorbilidad o el Índice de Enfermedades Co-Existentes ${ }^{45}$, ya que con éstos se puede controlar el sesgo de se- 
lección por tratamiento descrito en 1974, por Kaplan y Feinstein ${ }^{46}$ tanto en estudios prospectivos como retrospectivos.

Teniendo en cuenta la gran problemática del tema, otra opción es que se requiera la incorporación de modelos basados en la teoría de la complejidad o los análisis de niveles múltiples. Esta última aproximación es respuesta a un problema frecuente en los estudios epidemiológicos, resultante de considerar que todas las variables se deben medir a nivel individual, ignorando incluso que muchas pertenecen a niveles superiores de agregación (hogar, vecindario, municipio, por ejemplo $)^{47,48}$. La reproducibilidad de estudios sobre el tema, bajos nuevas y mejores consideraciones metodológicas, permitirán descubrir- de existir- el verdadero sentido y fuerza de esta asociación.

\section{Las potenciales implicaciones en salud pública}

Las geohelmintiasis son infecciones altamente prevalentes en el mundo en desarrollo que afectan especialmente a la población escolar entre 5 a 15 años ${ }^{1}$. Después de la malaria, explican más del $40 \%$ de la carga de la enfermedad por patologías tropicales siendo responsables de más de 39 millones de años perdidos ajustados por discapacidad cada año ${ }^{2}$. Los individuos infectados pueden padecer trastornos como anemia, desnutrición, mal desempeño escolar y trastornos del desarrollo y crecimiento ${ }^{49}$. A pesar de lo anterior, las geohelmintiasis son consideradas en la actualidad enfermedades desatendidas ya que, a pesar de su alta prevalencia y morbilidad asociada, ha disminuido el interés en su investigación y control ${ }^{50}$. El interés en estudiar los potenciales efectos de las geohelmintiasis sobre otras infecciones como malaria, tuberculosis yVIH, que impactan enormemente sobre la carga de la enfermedad, es motivado en parte por la necesidad sentida de repriorizar el control de las geohelmintiasis. La demostración de que los geohelmintos podrían aumentar el riesgo de incidencia de malaria tendría una repercusión sobre las políticas de control en los países en vía de desarrollo, ya que la distribución de antihelmínticos y el saneamiento ambiental serían considerados también como parte de estrategias transversales de control de la malaria.

Siendo clara la escasez de evidencia sobre las asociaciones entre los geohelmintos e incidencia de malaria como también sus serias limitaciones metodológicas, es preocupante que se hayan comenzado a hacer estimaciones serias de impacto en salud pública de una relación nada esclarecida. Estas consideraciones, además de ser apresuradas, podrían no justificarse. El control de las geohelmintiasis no debería estar supeditado a la existencia o no de una asociación con la incidencia malaria, ya que se dispone de estrategias de control de bajo costo y alta efectividad, lo cual se sabe puede tener un gran impacto en la carga de enfermedad de las poblaciones afectadas.

Ciertos estudios han sugerido también una asociación protectora entre la infección con geohelmintos, especialmenteA.lumbricoides, para el desarrollo de malaria cerebral ${ }^{51,52} \mathrm{y}$ falla renal aguda secundaria a malaria ${ }^{53}$; sin embargo, otro estudio más reciente ha encontrado una asociación totalmente opuesta ${ }^{54}$. La evidencia sobre este tema, al igual que la relacionada con incidencia de malaria, es todavía más escasa y divergente, además también tiene amplias limitaciones de validez. Pero de la misma manera, la existencia o no de una influencia de las geohelmintiasis en el desarrollo de malaria clínica no debería poner en duda el control de estas parasitosis, ya que existen determinantes bien conocidos mucho más importantes - del curso clínico y de la mortalidad de malaria - que lo que podrían ser las geohelmintiasis. Incluso en el caso de demostrarse un potencial efecto de los geohelmintos para el desarrollo de malaria complicada, esto lejos de poner en duda el control de las geohelmintisis reforzaría la necesidad de controlar simultáneamente ambas enfermedades.

Si bien el esclarecer la relación entre geohelmintiasis e incidencia de malaria es importante para la epidemiología, dada la relevancia que tienen estas enfermedades y 
que ya se conocen varios de sus determinantes proximales y distales, es recomendable que no se exagere la continuación de estudios de este tipo, fenómeno denominado "epidemiología circular" ${ }^{55}$, sino que más bien se enfatice la investigación en las acciones de prevención y control de los determinantes. Debe recordarse que el conocimiento sólo es uno de los componentes del "triángulo que mueve montañas" 56 , y que los movimientos sociales y la participación política también influyen en el alcance del bienestar de las poblaciones. Dada la evidencia sólida disponible para hacer prevención y control de la geohelmintiasis y la malaria urge ponerlas en marcha, sin esperar muchos más refinamientos en el conocimiento. Por otra parte, investigaciones profundas que indaguen sobre estrategias transversales para el control costo-efectivo y socialmente saludable de enfermedades con determinantes comunes como las geohelmintiasis y malaria, podría ser algo deseable mientras a la par se hacen esfuerzos, desde la comunidad científica, por impulsar la movilización social y la voluntad política. En este sentido, nuestro compromiso parece ser más complicado pero también quizá más importante.

\section{Referencias}

1. World Health Organization. Schistosomiasis and soil transmitted helminth infections. Wkly Epidemiol Rec 2006; 81: 145-64.

2. De Silva NR, Brooker S, Hotez PJ, Montresor A, Engels D, Savioli L. Soil transmitted helminth infections: updating the global picture. Trends Parasitol 2003; 19: 547-51.

3. World Health Organization. World malaria report 2005. Geneva:WHO; 2005.

4. Petney TN, Andrews RH. Multiparasite communities in animals and humans: frequency, structure and pathogenic significance. Int J Parasitol 1998; 28: 377-93.

5. Murray J, Murray A, Murray M, Murray C. The biological suppression of malaria: an ecological and nutritional interrelationship of a host and two parasites. Am J Clin Nutr 1978; 31: 1363-66.

6. Nacher M. Worms and malaria: noisy nuisances and silent benefits. Parasite Immunol 2002; 24: 391-3.

7. Basavaraju S, Schantz P. Soil-transmitted helminths and Plasmodium falciparum malaria: epidemiology, clinical manifestations, and the role of nitric oxide in malaria and geohelminth co-infection. Do worms have a protective role in P. falciparum infection? Mt Sinai J Med 2006; 73: 1098-104.

8. Druilhe P. Worms and malaria: mixing up clinical entities can only lead to confusion. Trends Parasitol 2006; 22: 351-2.

9. Mwangi TW. Bethony J, Brooker J. Malaria and helminthes interaction in humans: an epidemiologic viewpoint. Ann Trop Med Parasitol 2006; 100: 551-70.

10. Fenn B, Morris SS, Black RE. Comorbidity in childhood in northern Ghana: magnitude, associated factors, and impact on mortality. Int J Epidemiol 2005; 34:368-75.
11. Gijsen R, Hoeymans N, Schellevis FG, Ruwaard D, Satariano WA, van den Bos GAM. Causes and consequences of comorbidity: A review. J Clin Epidemiol 2001; 54: 661-74.

12. Feinstein AR. The pre-therapeutic classification of co-morbidity in chronic disease. J Chron Dis 1970; 23: 455-69.

13. Mulholland K. Commentary: comorbidity as a factor in child health and child survival in developing countries. Int J Epidemiol 2005; 34: 375-7.

14. U. S. Preventive Services Task Force. Guide to clinical preventive services, Ed 2, p 862. Baltimore: U. S. Preventive Services Task Force; 1996.

15. Brutus L, Watier L, Briand V, Hanitrasoamampionona V, Razanatsoarilala H, Cot M. Parasitic co-infections: does Ascaris lumbricoides protect against Plasmodium falciparum infection? Am J Trop Med Hyg 2006; 75:194-8.

16. Chen LY, Lin YL, Chiang BL. Levamisole enhances immune response by affecting the activation and maturation of human monocyte-derived dendritic cells. Clin Exp Immunol 2007 Nov 14; [Epub ahead of print])

17. Dondorp AM, Silamut K, Charunwatthana P, Chuasuwanchai S, Ruangveerayut R, krintratun S, et al. Levamisole inhibits sequestration of infected red blood cells in patients with falciparum malaria. J Infect Dis 2007; 196: 460-6.

18. Brutus L, Watier L, Briand V, Hanitrasoamampionona V, Razanatsoarilala H, Cot M. Confirmation of the protective effect of Ascaris lumbricoides on Plasmodium falciparum infection: results of a randomized trial in Madagascar. Am J Trop Med Hyg 2007;77: 1091-3 
19. Nacher M, Singhasivanon P, Yimsamran S, Manibunyong $\mathrm{W}$, Thanyavanich N, Wuthisen R, et al. Intestinal helminth infections are associated with increased incidence of Plasmodium falciparum malaria in Thailand. J Parasitol 2002; 88: 55-8.

20. Shapiro AE, Tukahebwa EM, Kasten J, Clarke SE, Magnussen P, Olsen A, et al. Epidemiology of helminth infections and their relationship to clinical malaria in southwest Uganda. Trans R Soc Trop Med Hyg 2005; 99: $18-24$.

21. Filmer D, Pritchett L. Estimating wealth effects without expenditure data-or tears: an application to educational enrollments in states of India. Demography 2000; 38: 115-32.

22. Spiegel A, Tall A, Raphenon G, Trape JF, Druilhe P. Increased frequency of malaria attacks in subjects co-infected by intestinal worms and Plasmodium falciparum malaria. Trans R Soc Trop Med and Hyg 2003; 97: 198-9.

23. Kazembe LN, Namangale JJ. A Bayesian multinomial model to analyse spatial patterns of childhood comorbidity in Malawi. Eur J Epidemiol 2007; 22: 545-56.

24. Yé Y, Hoshen M, Louis V, Séraphin S, I Traoré I, Sauerborn R. Housing conditions and Plasmodium falciparum infection: protective effect of iron-sheet roofed houses. Malar J 2006; 5: 8

25. Konradsen F, Amerasinghe P, Van Der Hoek W, Amerasinghe F, Perera D, Piyaratne M. Strong association between house characteristics and malaria vectors in Sri lanka. Am J Trop Med Hyg 2003; 68: 177-81.

26. Gunawardena DM, Wickremasinghe AR, Muthuwatta L, Weerasingha S, Rajakaruna J, Senanayaca T, et al. Malaria risk factors in an endemic region of Sri Lanka, and the impact and cost implications of risk factor-based interventions. Am J Trop Med Hyg 1998; 58: 533-42.

27. Gunawardena GS, Karunaweera ND, Ismail MM. Socio-economic and behavioural factors affecting the prevalence of Ascaris infection in a low-country tea plantation in Sri Lanka. Ann Trop Med Parasitol 2004; 98: 615-21.

28. Friedman JF, Kwena AM, Mirel LB, Kariuki SK, Terlouw DJ, Phillips-Howard PA, et al. Malaria and nutritional status among pre-school children: results from crosssectional surveys in western Kenya. Am J Trop Med. Hyg 2005; 73: 698-704.

29. Schaible UE, Kaufmann SH. Malnutrition and Infection: Complex mechanisms. PLoS Med 2007; 4 : 806-12.

30. Perez JM. Nutritional status and the IgE response against Ascaris lumbricoides in children from a tropical slum. Trans R Soc Trop Med Hyg 1995; 89: 562-5.

31. Hagel I, Lynch NR, Puccio F, Rodríguez O, Luzondo R, Rodríguez P, et al. Defective regulation of the protective IgE response against intestinal helminth Ascaris lumbricoides in malnourished children. J Trop Pediatr 2003; 49:1 36-42.
32. Caulfield L, Richard S, Black R. Undernutrition as an underlying cause of malaria morbidity and mortality in children less than five years old. Am J Trop Med Hyg 2004; 71: 55-63.

33. Brooker S,Clarke S, Njagi JK, Polack S, Mugo B, Estambale B, et al. Spatial clustering of malaria and associated risk factors during an epidemic in a highland area of western Kenya. Trop Med Int Health 2004; 9: 757 66.

34. Erhart A, Thang ND, Ky PV, Tinh TT, Overmeir CV, Speybroeck N, et al. Epidemiology of forest malaria in central Vietnam: a large scale cross-sectional survey. Malar J 2005; 4: 58.

35. Habbari K, Tifnouti A, Bitton G, Mandil A. Geohelmintic infections associated with raw wastewater reuse for agricultural purposes in Beni-Mellal, Morroco. Parasitol Int 2000; 48: 249-54.

36. Sintasath DM, Ghebremeskel T, Lynch M, Kleinau E , Bretas G, Shililu J, et al. Malaria prevalence and associated risk factors in Eritrea. Am J Trop Med Hyg 2005; 72: 682-7.

37. Booth $\mathrm{M}$. The role of residential location in apparent helminth and malaria associations. Trends Parasitol 2006; 22: 359-62.

38. Hartgers FC, Yazdanbakhsh M. Co-infection of helminths and malaria: modulation of the immune responses to malaria. Parasite Immunol 2006; 28: 497-506.

39. Van Riet E, Hartgers FC, Yazdanbakhsh M. Chronic helminth infections induce immunomodulation: Consequences and mechanisms. Immunobiology 2007; 212:475-90.

40. Garraud O, Mahanty S, Perrault R. Malaria-specific antibody subclasses in immune individuals: a key source of information for vaccine design. Trend in Immunol $2003 ; 24: 30-5$

41. Naus CW, Jones FM, Satti MZ, Joseph S, Riley EM, Kimani G, et al. Serological responses among individuals in areas where both schistosomiasis and malaria are endemic: cross-reactivity between Schistosoma mansoni and Plasmodium falciparum. J Infect Dis 2003; 187: 1272-82.

42. Correa-Oliveira R, Dusse LM, Viana IR, Colley DG, Santos Carvalho O, Gazzinelli G. Human antibody responses against schistosomal antigens. I. Antibodies from patients with Ancylostoma, Ascaris lumbricoides or Schistosoma mansoni infections react with schistosome antigens. Am J Trop Med Hyg 1988; 38: 348-55.

43. VanderWeele TJ, Robins JM. The identification of synergism in the sufficient-component-cause framework. Epidemiology 2007; 18: 329-39.

44. Darroch J. Biologic synergism and parallelism. Am J Epidemiol 1997; 145: 661-8. 
45. Hall SF. A user's guide to selecting a comorbidity index for clinical research. J Clin Epidemiol 2006; 59: 849-55.

46. Kaplan MH, Feinstein AR. The importance of classifying initial co-morbidity in evaluating the outcome of diabetes mellitus. J Chron Dis 1974; 27: 387-404.

47. Duncan C, Jones K, Moon G. Context, composition and heterogeneity: Using multilevel models in health research. Soc Sci Med 1998; 46: 97-117.

48. Oakes JM. The (mis)estimation of neighborhood effects: causal inference for a practicable social epidemiology. Soc Sci Med 2004; 58: 1929-52.

49. Cromptom DW, Curtale F, Pezzotti P, Saad YS, Aloi A. How much human helminthiasis is there in the world? $J$ Parasitol 1999; 85: 397-403.

50. .World Health Organization. Global plan to combat neglected tropical diseases 2008-2015. Geneve: WHO; 2007.

51. Nacher M, Singhasinovanon S, Treeprasertzuk S, Vannaphan S, Traore B, Looareesuwan S, et al. Intestinal helminths and malnutrition are independently associated with protection from cerebral malaria in Thailand. Ann Trop Med Parasitol 2002; 96; 5-13.
52. Nacher M, Gay F, Singhasinovanon P, Krudsood S, Treeprasertzuk S, Mazier D, et al. Ascaris lumbricoides is associated with protection from cerebral malaria. Parasite Inmmunol 2000; 22: 107-13.

53. Nacher P, Singhasinovanon P, Silachamroon U, Treeprasertzuk S, Vannaphan S, Traore B, et al. Helminth infections are associated with protection from malariarelated acute renal failure and jaundice in Thailand. Am J Trop Med Hyg 2001; 65: 834-6.

54. Le Hesran JY, Akiana J, Ndiaye el HM, Día M, Senghor P, Konate L. Severe malaria attack is associated with high prevalence of Ascaris lumbricoides infection among children in rural Senegal. Trans R Soc Trop Med Hyg 2004; 98: 397-9.

55. Kuller LH. Circular epidemiology. Am J Epidemiol 1999; 150: 897-903.

56. Wasi P. "Triangle that moves the mountain" and health systems reform movement in Thailand. Human Resources Health Develop J 2000; 4: 106-10.

Recebido em: 23/11/07

Versão final reapresentada em: 17/06/08

Aprovado em: 25/06/08 